\title{
Choice of the regularization parameter for the Cauchy problem for the Laplace equation
}

\author{
Magda Joachimiak \\ Faculty of Environmental Engineering and Energy, \\ Poznan University of Technology, Poznan, Poland
}

\begin{abstract}
Purpose - In this paper, the Cauchy-type problem for the Laplace equation was solved in the rectangular domain with the use of the Chebyshev polynomials. The purpose of this paper is to present an optimal choice of the regularization parameter for the inverse problem, which allows determining the stable distribution of temperature on one of the boundaries of the rectangle domain with the required accuracy.

Design/methodology/approach - The Cauchy-type problem is ill-posed numerically, therefore, it has been regularized with the use of the modified Tikhonov and Tikhonov-Philips regularization. The influence of the regularization parameter choice on the solution was investigated. To choose the regularization parameter, the Morozov principle, the minimum of energy integral criterion and the L-curve method were applied.

Findings - Numerical examples for the function with singularities outside the domain were solved in this paper. The values of results change significantly within the calculation domain. Next, results of the sought temperature distributions, obtained with the use of different methods of choosing the regularization parameter, were compared. Methods of choosing the regularization parameter were evaluated by the norm $N_{\max }$.

Practical implications - Calculation model described in this paper can be applied to determine temperature distribution on the boundary of the heated wall of, for instance, a boiler or a body of the turbine, that is, everywhere the temperature measurement is impossible to be performed on a part of the boundary.

Originality/value - The paper presents a new method for solving the inverse Cauchy problem with the use of the Chebyshev polynomials. The choice of the regularization parameter was analyzed to obtain a solution with the lowest possible sensitivity to input data disturbances.
\end{abstract}

Keywords Inverse Cauchy problem, L-curve, Minimum of energy integral criterion, Morozov principle, Regularization parameter, Laplace's equation, Regularization

Paper type Research paper

\section{Nomenclature}

$a \quad=$ multinomial coefficient of the function of distribution of temperature $\tilde{T}(w)$;

$c \quad=$ multinomial coefficient of the function of distribution of temperature $T(x, y)$;

$E(\alpha) \quad=$ functional, energy integral;

$J_{\alpha} \quad=$ regularizing functional $\left(J_{\alpha}=J+\alpha^{2} I\right)$;

$m \quad$ = number of Chebyshev nodes on the $y$-axis;

$n \quad=$ number of Chebyshev nodes on the $x$-axis;

$N_{1}-1 \quad=$ degree of the polynomial describing unknown distribution of temperature on the $\Gamma_{1}$ boundary;

(C) Magda Joachimiak. Published by Emerald Publishing Limited. This article is published under the Creative Commons Attribution (CC BY 4.0) licence. Anyone may reproduce, distribute, translate and create derivative works of this article (for both commercial and non-commercial purposes), subject to full attribution to the original publication and authors. The full terms of this licence may be seen at http://creativecommons.org/licences/by/4.0/legalcode

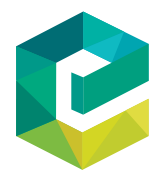

International Journal of Numerical Methods for Heat \& Fluid Flow Vol. 30 No. 10,2020 pp. $4475-4492$ Emerald Publishing Limited Emerald Publishing Limited DOI 10.1108/HFF-10-2019-0730 
$N_{\max }=$ norm;

$q \quad=$ heat flux density, $\mathrm{W} / \mathrm{m}^{2} \mathrm{~K}$;

$T \quad=$ temperature, $\mathrm{K}$;

$\tilde{T} \quad=$ temperature, function dependent on the Chebyshev node;

$w \quad=$ Chebyshev node;

$W_{i} \quad=$ Chebyshev polynomial of the first kind of $i$-th degree;

$x, y \quad=$ Cartesian coordinates;

$[x]_{n} \quad=$ integer part of the division of number $x$ by $n$; and

$x \bmod n=$ remainder of the division of number $x$ by $n$.

Greek symbols

$\alpha=$ regularization parameter;

$\delta$ = error;

$\delta_{M}=$ error of measurement data (Morozov principle);

$\gamma=$ multinomial coefficient, pertains to the sought temperature distribution on the boundary $\Gamma_{1}$;

$\Gamma \quad=$ boundary of the domain $\Omega,\left(\Gamma=\Gamma_{1} \cup \Gamma_{2} \cup \Gamma_{3} \cup \Gamma_{4}\right)$; and

$\Omega=$ calculation domain.

\section{Subscript}

A = analytical solution;

$\mathrm{c}$ = calculated value;

$\mathrm{m}$ = measured value;

ran = randomly disturbed value; and

$\Gamma_{i}=$ on the boundary $\Gamma_{i}($ for $i=1,2,3$ and 4$)$.

\section{Introduction}

Inverse problems are ill-posed in the Hadamard (1902) sense. It means that a slight disturbance to measurement data results in significant errors of the obtained results (Joachimiak and Ciałkowski, 2017, 2014, 2018; Nowak, 2017). Therefore, problems of such type need to be regularized. There are many methods used to regularize inverse problems. Among them, there is the Tikhonov regularization (Beck and Woodbury, 2016; Chen et al, 2019; Djerrar et al, 2017; Frąckowiak et al., 2019a; Laneev, 2018; Marin, 2010, 2016; Niu et al., 2014; Sun, 2016; Tikhonov and Arsenin, 1977; Yaparova, 2016), the Tikhonov-Philips regularization (Joachimiak et al, 2019a), the discrete Fourier transform (Frąckowiak and Ciałkowski, 2018; Wróblewska et al., 2015) and SVD algorithm (Hasanov and Mukanova, 2015). In her article, Cheruvu (2017) applied the wavelet regularization of Laplace's equation in the arbitrarily shaped domain. The solution to the Cauchy problem for the Laplace's equation was also sought with the use of the iterative Tikhonov-type method (Delvare and Cimetière, 2017). Han et al. (2011) in the article presented numerical tests concerning the solution to the Cauchy problem for Laplace's equation with the use of the energy regularization method. Obtained results were compared with the Tikhonov regularization for which the regularization parameter was chosen based on the Morozov principle. In the paper of Liu and Wang (2018), the Cauchy problem for the Laplace's equation was solved with the use of the method of fundamental solutions and the energy regularization technique to choose the source points. The Laplace's equation was also solved with the use of iterative algorithms (Frąckowiak et al., 2015a, 2015b), of the Trefftz method (Ciałkowski and Frąckowiak, 2002; Ciałkowski and Grysa, 2010; Grysa et al., 2012; Hożejowski, 2016; Lin et al., 2018), of the method of fundamental solution (Kołodziej and Mierzwiczak, 2008; Mierzwiczak et al, 2015; Mierzwiczak and Kołodziej, 2011) and of the collocation method (Joachimiak et al, 2016). In many cases, the regularization of the inverse problem concerns the 
problem of choosing the regularization parameter. The regularization parameter can be chosen based on the Morozov principle (Chen et al., 2019; Han et al., 2011; Joachimiak et al., 2019a; Marin, 2016; Morozov, 1984; Sun, 2016) or using the L-curve method (Jin and Zheng, 2006; Marin and Munteanu, 2010; Marin, 2005). In the study of Marin (2011), the optimal regularization parameter was sought based on the generalized cross-validation criterion. Currently, research work focuses on finding new methods of regularization (Cheng and Feng, 2014; Zhuang and Chen, 2017) and on the modification of already known and used methods (Yang et al., 2015; Zheng and Zhang, 2018). Because of a wide application of inverse problems in engineering problems, such as the cooling of the blades in gas turbines (Frackowiak et al., 2017; Frąckowiak et al., 2019b; Frąckowiak et al., 2011), analysis of the boiling heat transfer in minichannels (Hożejowska et al., 2009; Maciejewska and Piasecka, 2017), analysis of thermal and thermo-chemical treatment (Joachimiak et al., 2019b) or monitoring of power boilers operation (Taler et al., 2016, 2017), developing methods for regularization of these problems and investigating the process of choosing the regularization parameter are very significant.

In this article, the solution to the Cauchy problem for the Laplace's equation was investigated with the use of the Chebyshev polynomials. To regularize the solution to the inverse problem, the modification of the Tikhonov and of the Tikhonov-Philips regularizations, described in the article (Joachimiak et al., 2019a) was applied. The choice of the regularization parameter was made based on the Morozov principle, the minimum energy integral criterion and the L-curve method.

\section{Calculation model}

In many technical applications, it is impossible to measure temperature on the boundary of the heated component of the device or machine, such as the combustion chamber, the inner side of the body of a turbine or a boiler. Then, the distribution of temperature can be determined by finding the solution to the inverse problem. Based on the distribution of temperature on the part of the boundary $\left(T_{\Gamma_{2}}, T_{\Gamma_{3}}, T_{\Gamma_{4}}\right.$, fig. 1) and, additionally, knowing the heat flux density on the part of the boundary $\left(q_{\Gamma_{3}}\right.$, fig. 1$)$ one can determine the distribution of temperature on the boundary, where it is impossible to measure this temperature $\left(T_{\Gamma_{1}}\right.$, fig. 1$)$. Such a posed problem is the Cauchy problem, particularly sensitive to errors in measurement and in the calculation. In the stationary thermal field, the heat equation is reduced to the Laplace's equation (for the non-linear case, the Kirchhoff's substitution transforms the heat equation into the Laplace's equation).

$$
\frac{\partial^{2} T}{\partial x^{2}}+\frac{\partial^{2} T}{\partial y^{2}}=0
$$

Laplace's equation is solved in the domain $\Omega=\left\{(x, y) \in \mathbb{R}^{2}:-1 \leq x \leq 1,-1 \leq y \leq 1\right\}$ with the following boundary conditions (Figure 1):

$$
\begin{gathered}
T(x, y=1)=T_{\Gamma_{2}}(x) \text { for }-1 \leq x \leq 1 \\
T(x=-1, y)=T_{\Gamma_{3}}(y) \text { for }-1 \leq y \leq 1 \\
\frac{\partial T(x=-1, y)}{\partial n}=q_{\Gamma_{3}}(y) \text { for }-1 \leq y \leq 1 \\
T(x, y=-1)=T_{\Gamma_{4}}(x) \text { for }-1 \leq x \leq 1
\end{gathered}
$$


HFF

30,10

4478

It was assumed that the solution can be noted as the linear combination of the Chebyshev polynomials (Paszkowski, 1975).

$$
T\left(x_{i}, y_{j}\right)=\sum_{p=0}^{n-1} \sum_{q=0}^{m-1} c_{p q} W_{p}\left(x_{i}\right) W_{q}\left(y_{j}\right)
$$

To solve the Cauchy problem, the collocation method was used. It was assumed that there are $n$ points along the $x$-axis and $\mathrm{m}$ points along the $y$-axis (including points on the boundary). Collocation points being inside the interval $(-1,1)$ are the Chebyshev nodes (Paszkowski, 1975). Nodes were renumbered, which enables the temperature function to be noted in the following equation (7):

$$
\tilde{T}\left(w_{l}\right)=\sum_{k=1}^{m n} a_{k} W_{[k-1]_{m}}\left(x_{(l-1) \bmod n+1}\right) W_{(k-1) \bmod m}\left(y_{[l-1]_{n}+1}\right)
$$

where the coefficients $a_{k}(k=1,2, \ldots, m n)$ are unknown. Sought temperature distribution on the boundary $\Gamma_{1}$ was assumed as the linear combination of the Chebyshev polynomials (Paszkowski, 1975).

$$
T_{\Gamma_{1}}(y)=\sum_{h=1}^{N_{1}} \gamma_{h} W_{h-1}(y)
$$

Coefficients $a_{k}(k=1,2, \ldots, m n)$ are expressed by the values of coefficients $\gamma_{i}(i=1,2, \ldots$, $\left.N_{1}\right)$. Hence, the determination of the temperature distribution is reduced to the determination of coefficients $\gamma_{i}$. To do so, the functional of the following form was minimized.

$$
\begin{aligned}
J= & \int_{\Gamma_{2}}\left(T_{c, \Gamma_{2}}-T_{m, \Gamma_{2}}\right)^{2} d \Gamma_{2}+\int_{\Gamma_{3}}\left(T_{c, \Gamma_{3}}-T_{m, \Gamma_{3}}\right)^{2} d \Gamma_{3}+\int_{\Gamma_{3}}\left(\frac{\partial T_{c, \Gamma_{3}}}{\partial n}-q_{m, \Gamma_{3}}\right)^{2} d \Gamma_{3} \\
& +\int_{\Gamma_{4}}\left(T_{c, \Gamma_{4}}-T_{m, \Gamma_{4}}\right)^{2} d \Gamma_{4}
\end{aligned}
$$

where $\mathrm{c}$ in subscript denotes the calculated value, while $\mathrm{m}$ denotes the measured value. The integral in equation (9) on the boundary $\Gamma_{2}$ can be noted in the following equation (10):

Figure 1.

Calculation domain

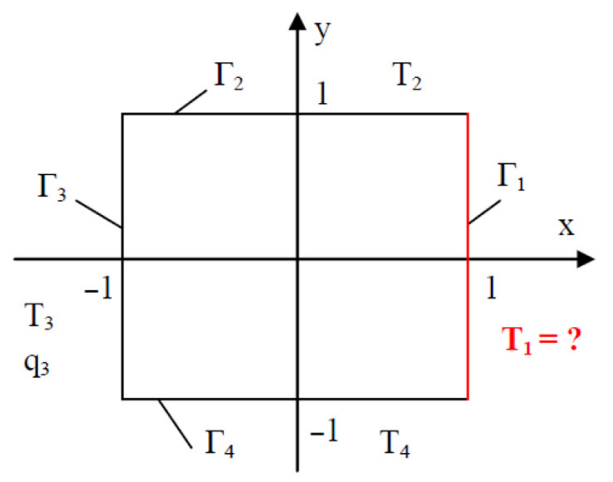




$$
J_{\Gamma_{2}}=\int_{\Gamma_{2}}\left(T_{c, \Gamma_{2}}-T_{m, \Gamma_{2}}\right)^{2} d \Gamma_{2}=\sum_{i=1}^{n} \int_{\Gamma_{2 i}}\left(T_{c, \Gamma_{2 i}}-T_{m, \Gamma_{2 i}}\right)^{2} d \Gamma_{2 i}
$$

Applying numerical integration we have:

Cauchy problem for the Laplace equation

$$
J_{\Gamma_{2}}=\sum_{i=1}^{n} \Delta x_{i}\left(T_{c}\left(x_{i}, 1\right)-T_{m}\left(x_{i}, 1\right)\right)^{2}=\sum_{i=1}^{n}\left(\sqrt{\Delta x_{i}}\left(T_{c}\left(x_{i}, 1\right)-T_{m}\left(x_{i}, 1\right)\right)\right)^{2}
$$

where $\Delta x_{1}=\frac{x_{2}-x_{1}}{2}, \Delta x_{i}=\frac{x_{i+1}-x_{i-1}}{2}$ for $i=2,3, \ldots, n-1$ and $\Delta x_{n}=\frac{x_{n}-x_{n-1}}{2}$. Having inserted the equation (7) into the equation (11), we obtained:

$$
J_{\Gamma_{2}}=\sum_{i=1}^{n}\left(\sqrt{\Delta x_{i}}\left(\sum_{k=1}^{m n} a_{k} W_{[k-1]_{m}}\left(x_{i}\right) W_{(k-1) \bmod m}(1)-T_{m}\left(x_{i}, 1\right)\right)\right)^{2}
$$

Solving the direct problem, where the temperature on boundaries $\Gamma_{1}, \Gamma_{2}, \Gamma_{3}$ and $\Gamma_{4}$ was known, was reduced to solving the matrix equation

$$
A x=b
$$

what was described in detail in the paper (Joachimiak et al., 2019a). Based on the solution to the direct problem, constants $a_{k}$ [Equation (12)] are of the following equation (14):

$$
a_{k}=F_{k}+\sum_{h=1}^{N_{1}} \gamma_{h} H_{h, k}
$$

where $H_{h, k}=\sum_{j=2}^{m-1} \tilde{A}_{k, j n} W_{h-1}\left(y_{j}\right)$, while $\tilde{A}_{k, j n}(k=1,2, \ldots, m n ; j=2,3, \ldots, m-1)$ are elements of the matrix $A^{-1}$. After substituting equation (14) into equation (12) we obtained:

$$
\begin{aligned}
J_{\Gamma_{2}}=\sum_{i=1}^{n}( & \left.\sqrt{\Delta x_{i}}\left(\sum_{k=1}^{m n}\left(F_{k}+\sum_{h=1}^{N_{1}} \gamma_{h} H_{h, k}\right) W_{[k-1]_{m}}\left(x_{i}\right) W_{(k-1) \bmod m}(1)-T_{m}\left(x_{i}, 1\right)\right)\right)^{2} \\
= & \sum_{i=1}^{n}\left(\sum_{h=1}^{N_{1}} \gamma_{h} \sum_{k=1}^{m n} \sqrt{\Delta x_{i}} H_{h, k} W_{[k-1]_{m}}\left(x_{i}\right) W_{(k-1) \bmod m}(1)\right. \\
& \left.+\sum_{k=1}^{m n} \sqrt{\Delta x_{i}} F_{k} W_{[k-1]_{m}}\left(x_{i}\right) W_{(k-1) \bmod m}(1)-T_{m}\left(x_{i}, 1\right)\right)^{2} \\
& =\sum_{i=1}^{n}\left(\sum_{h=1}^{N_{1}} \gamma_{h} C_{1}(i, h)-D_{1}(i)\right)^{2}
\end{aligned}
$$

We would like the integral $J_{\Gamma_{2}}$ to have a value equal to zero or as close to zero as possible, hence, we equate the squared expression [Equation (15)] to zero. Hence, we have that: 
$\mathrm{HFF}$

30,10

$$
\underset{i=1,2, \ldots, n}{\forall} \sum_{h=1}^{N_{1}} \gamma_{h} C_{1}(i, h)=D_{1}(i)
$$

We obtain $n$ linear equations of the following equation (17):

$$
\left\{C_{1}(i, h)\right\}\left\{\gamma_{h}\right\}=D_{1}(i)
$$

for $i=1,2, \ldots, n$ and $h=1,2, \ldots, N_{1}$. It can be reduced to the matrix equation.

$$
\left[C_{1, n}\right]\{\gamma\}=\left\{D_{1, n}\right\}
$$

Similarly, for other integrals [Equation (9)] we obtained:

$$
\begin{gathered}
J_{\Gamma_{3}}=\int_{\Gamma_{3}}\left(T_{c, \Gamma_{3}}-T_{m, \Gamma_{3}}\right)^{2} d \Gamma_{3}=\sum_{i=1}^{m}\left(\sum_{h=1}^{N_{1}} \gamma_{h} C_{2}(i, h)-D_{2}(i)\right)^{2} \\
J_{q, \Gamma_{3}}=\int_{\Gamma_{3}}\left(\frac{\partial T_{c, \Gamma_{3}}}{\partial n}-q_{m, \Gamma_{3}}\right)^{2} d \Gamma_{3}=\sum_{i=1}^{m}\left(\sum_{h=1}^{N_{1}} \gamma_{h} C_{3}(i, h)-D_{3}(i)\right)^{2} \\
J_{\Gamma_{4}}=\int_{\Gamma_{4}}\left(T_{c, \Gamma_{4}}-T_{m, \Gamma_{4}}\right)^{2} d \Gamma_{4}=\sum_{i=1}^{n}\left(\sum_{h=1}^{N_{1}} \gamma_{h} C_{4}(i, h)-D_{4}(i)\right)^{2}
\end{gathered}
$$

After $J_{\Gamma_{3}}, J_{q, \Gamma_{3}}$ and $J_{\Gamma_{4}}$ had been equated to zero, equations of the following forms were obtained:

$$
\begin{gathered}
\underset{i=1,2, \ldots, m}{\forall} \sum_{h=1}^{N_{1}} \gamma_{h} C_{2}(i, h)=D_{2}(i) \\
\underset{i=1,2, \ldots, m}{\forall} \sum_{h=1}^{N_{1}} \gamma_{h} C_{3}(i, h)=D_{3}(i) \\
\underset{i=1,2, \ldots, n}{\forall} \sum_{h=1}^{N_{1}} \gamma_{h} C_{4}(i, h)=D_{4}(i)
\end{gathered}
$$

Based on equations (16) and (22)-(24), an oversized system of linear equations was obtained as the matrix equation, which would be solved with the use of the SVD algorithm: 


$$
\left[\begin{array}{l}
{\left[C_{1, n}\right]} \\
{\left[C_{2, m}\right]} \\
{\left[C_{3, m}\right]} \\
{\left[C_{4, n}\right]}
\end{array}\right]\{\gamma\}=\left\{\begin{array}{c}
\left\{D_{1, n}\right\} \\
\left\{D_{2, m}\right\} \\
\left\{D_{3, m}\right\} \\
\left\{D_{4, n}\right\}
\end{array}\right\}
$$

Cauchy problem for the Laplace equation

what can be noted in the shorter form:

$$
[B M]\{\gamma\}=\{B W\}
$$

Because of a great sensitivity of results to disturbances to measurement data, the Cauchy problem was regularized. The regularizing functional of the following form was assumed:

$$
J_{\alpha}=J(\gamma)+\alpha^{2} I(\gamma)=\|[B M]\{\gamma\}-\{B W\}\|^{2}+\alpha^{2} \int_{\Gamma_{1}}\left((\tilde{T})^{2}+\left(\frac{\partial \tilde{T}}{\partial y}\right)^{2}\right) d \Gamma_{1}
$$

Regularization term can be noted as the sum of integrals.

$$
\alpha^{2} I(\gamma)=\alpha^{2} \int_{\Gamma_{1}}\left((\tilde{T})^{2}+\left(\frac{\partial \tilde{T}}{\partial y}\right)^{2}\right) d \Gamma_{1}=\alpha^{2} \sum_{i=1}^{m-1} \int_{\Gamma_{1 i}}\left((\tilde{T})^{2}+\left(\frac{\partial \tilde{T}}{\partial y}\right)^{2}\right) d \Gamma_{1 i}
$$
where $\Gamma_{1}=\bigcup_{i=1}^{m-1} \Gamma_{1 i}$. Performing numerical integration using the trapezoidal rule, we
obtained:

$$
\alpha^{2} I(\gamma)=\sum_{i=1}^{m-1} \alpha^{2} \frac{y_{i+1}-y_{i}}{2}\left[\left(\tilde{T}\left(1, y_{i+1}\right)\right)^{2}+\left(\frac{\partial \tilde{T}\left(1, y_{i+1}\right)}{\partial y}\right)^{2}+\left(\tilde{T}\left(1, y_{i}\right)\right)^{2}+\left(\frac{\partial \tilde{T}\left(1, y_{i}\right)}{\partial y}\right)^{2}\right]
$$

On the boundary $\Gamma_{1}$ we have:

$$
\begin{gathered}
\tilde{T}\left(1, y_{i}\right)=\sum_{k=1}^{m n} a_{k} W_{[k-1]_{m}}(1) W_{(k-1) \bmod m}\left(y_{i}\right)=\sum_{k=1}^{m n}\left(F_{k}+\sum_{h=1}^{N_{1}} \gamma_{h} H_{h, k}\right) W_{[k-1]_{m}}(1) W_{(k-1) \bmod m}\left(y_{i}\right)= \\
=\sum_{k=1}^{m n} F_{k} W_{[k-1]_{m}}(1) W_{(k-1) \bmod m}\left(y_{i}\right)+\sum_{h=1}^{N_{1}} \gamma_{h} \sum_{k=1}^{m n} H_{h, k} W_{[k-1]_{m}}(1) W_{(k-1) \bmod m}\left(y_{i}\right)= \\
=A_{1}(i)+\sum_{h=1}^{N_{1}} \gamma_{h} A_{2}(i, h)
\end{gathered}
$$

$$
\begin{gathered}
\frac{\partial \tilde{T}\left(1, y_{i}\right)}{\partial y}=\sum_{k=1}^{m n} F_{k} W_{[k-1]_{m}}(1) W_{(k-1) \bmod m}^{\prime}\left(y_{i}\right)+\sum_{h=1}^{N_{1}} \gamma_{h} \sum_{k=1}^{m n} H_{h, k} W_{[k-1]_{m}}(1) W_{(k-1) \bmod m}^{\prime}\left(y_{i}\right)= \\
=A_{3}(i)+\sum_{h=1}^{N_{1}} \gamma_{h} A_{4}(i, h)
\end{gathered}
$$

Hence, 


$$
\begin{aligned}
\alpha^{2} I(\gamma)= & \sum_{i=1}^{m-1}\left\{\alpha \sqrt{\frac{y_{i+1}-y_{i}}{2}}\left(A_{1}(i+1)+\sum_{h=1}^{N_{1}} \gamma_{h} A_{2}(i+1, h)\right)\right\}^{2} \\
& +\sum_{i=1}^{m-1}\left\{\alpha \sqrt{\frac{y_{i+1}-y_{i}}{2}}\left(A_{3}(i+1)+\sum_{h=1}^{N_{1}} \gamma_{h} A_{4}(i+1, h)\right)\right\}^{2} \\
& +\sum_{i=1}^{m-1}\left\{\alpha \sqrt{\frac{y_{i+1}-y_{i}}{2}}\left(A_{1}(i)+\sum_{h=1}^{N_{1}} \gamma_{h} A_{2}(i, h)\right)\right\}^{2} \\
& +\sum_{i=1}^{m-1}\left\{\alpha \sqrt{\frac{y_{i+1}-y_{i}}{2}}\left(A_{3}(i)+\sum_{h=1}^{N_{1}} \gamma_{h} A_{4}(i, h)\right)\right\}^{2}
\end{aligned}
$$

Each of the components [Equation (32)] was equated to zero. The equation of the following form was obtained:

$$
\begin{gathered}
\underset{i=1,2, \ldots, m-1}{\forall} \alpha \sqrt{\frac{y_{i+1}-y_{i}}{2}}\left(A_{1}(i+1)+\sum_{h=1}^{N_{1}} \gamma_{h} A_{2}(i+1, h)\right)=0 \\
\underset{i=1,2, \ldots, m-1}{\forall} \alpha \sqrt{\frac{y_{i+1}-y_{i}}{2}}\left(A_{3}(i+1)+\sum_{h=1}^{N_{1}} \gamma_{h} A_{4}(i+1, h)\right)=0 \\
\underset{\substack{i=1,2, \ldots, m-1 \\
\forall}}{\forall} \alpha \sqrt{\frac{y_{i+1}-y_{i}}{2}}\left(A_{1}(i)+\sum_{h=1}^{N_{1}} \gamma_{h} A_{2}(i, h)\right)=0 \\
\underset{i=1,2, \ldots, m-1}{\forall} \alpha \sqrt{\frac{y_{i+1}-y_{i}}{2}}\left(A_{3}(i)+\sum_{h=1}^{N_{1}} \gamma_{h} A_{4}(i, h)\right)=0
\end{gathered}
$$

Equations (33)-(36) can be reduced to the following system of equations.

$$
\alpha[C M]\{\gamma\}=-\alpha\{C W\}
$$

where $\alpha$ is the regularization parameter. When the equation (26) and regularization [Equation (37)] are included, the following system of equations is obtained:

$$
\left[\begin{array}{c}
{[B M]} \\
\alpha[C M]
\end{array}\right]\{\gamma\}=\left\{\begin{array}{c}
\{B W\} \\
-\alpha\{C W\}
\end{array}\right\}
$$

The solution to the system of equations (38) was sought in the least-squares sense with the use of the SVD algorithm. 


\section{Choice of the regularization parameter}

To determine unknown regularization parameter $\alpha$, the Morozov principle, the minimum of energy integral criterion and L-curve method were applied. For the solution obtained with the use of the Morozov principle, the mean (Morozov_A) and the maximal (Morozov_B) errors of the heat flux density $\delta_{M}$ on the boundary $\Gamma_{3}$ were evaluated. Interval halving method was used to determine zero of the function $F_{M}(\alpha)$ defined by the following equation (39):

$$
\|[B M]\{\gamma\}-\{B W\}\|^{2}-\delta_{M}^{2}=F_{M}
$$

Unknown regularization parameter $\alpha$ was also sought based on the minimization of the functional (energy integral) of the following equation (40):

$$
E(\alpha)=\int_{\Omega}(\nabla T(\alpha))^{2} d \Omega, T(\alpha) \in C^{2}(\Omega)
$$

where $(\nabla T(\alpha))^{2}=\left(\frac{\partial T(\alpha)}{\partial x}\right)^{2}+\left(\frac{\partial T(\alpha)}{\partial y}\right)^{2}$. The minimum of the energy integral corresponds to satisfying the Laplace's equation (with respective boundary conditions), which is discussed in the paper (Gelfand and Fomin, 1979). Therefore, we choose the parameter $\alpha$ for which $\min _{\alpha} E(\alpha)$ occurs, i.e. the derivative $E^{\prime}=\frac{d E}{d \alpha}$ reverses the sign. Domain $\Omega$ was divided into rectangular domains with the use of equidistant nodes and next into domains being right-angled triangles. The integral value was calculated with the use of the finite element method. Value $\nabla T$ was determined based on the form of the solution equation (6). Values $\gamma_{h}$ were obtained by solving the equation (38).

On the basis of the solution of the equation (38), the L-curve was drawn as the correlation between $\|[B M]\{\gamma\}-\{B W\}\|$ on the $x$-axis and $\|[C M]\{\gamma\}-\{C W\}\|$ on the $y$-axis. We sought for the regularization parameter $\alpha$ with which corresponded the point of the L-curve locating on the curvature of this line. To evaluate the choice of the regularization parameter $\alpha$, the following norm was defined:

$$
N_{\max }=\frac{\max \left|T_{\Gamma 1 \_C}-T_{\Gamma 1 \_A}\right|}{\max \left|T_{\Gamma 1 \_A}\right|}
$$

\section{Numerical examples}

Calculations were made in the domain $\Omega$ for the function.

$$
f_{1}=\ln \left((x-a)^{2}+(y-b)^{2}\right), q_{1, \Gamma_{3}}=\frac{2(-1-a)}{(-1-a)^{2}+(y-b)^{2}}
$$

and

$$
f_{2}=\operatorname{Re}\left(\frac{1}{z-(a+b i)}\right)=\frac{x-a}{(x-a)^{2}+(y-b)^{2}}, q_{2, \Gamma_{3}}=\frac{-(-1-a)^{2}+(y-b)^{2}}{\left[(-1-a)^{2}+(y-b)^{2}\right]^{2}}
$$

Cauchy problem for the Laplace equation

4483 
HFF

30,10

4484

Table I.

Values of the norm $N_{\text {max }}$ for calculations without regularization $(\alpha=$ $0)$, without disturbance $\left(\delta_{\text {ran }}=\right.$ 0 ) and with random disturbance to the heat flux density up to $0.01 q\left(\delta_{\text {ran }}=0.01\right)$ and $0.02 q\left(\delta_{\text {ran }}=\right.$ 0.02)
We assumed such values of constants $a$ and $b$ that singularities would be outside the calculation domain $\Omega$ and that the values of gradients would change significantly within this domain $(a=1.3, b=1.3, b=1.1)$. Values of the norm $N_{\max }$ [Equation (41)], not including the regularization [Equation (26)], without disturbance $\left(\delta_{\text {ran }}=0\right)$ and with random disturbance to the heat flux density up to $0.01 q\left(\delta_{\text {ran }}=0.01\right)$ and to $0.02 q$ $\left(\delta_{\text {ran }}=0.02\right)$ are summarized in Table I. Disturbance $q$ is an additive function with the uniform distribution. A slight disturbance to measurement data results in a significant error of the sought temperature on the boundary $\Gamma_{1}$. Hence, it is necessary to regularize the inverse problem [Equation (38)] and to choose the regularization parameter $\alpha$ properly.

\subsection{Example 1}

Calculations were made for the function $f_{1}$ [Equation (42)]. Heat flux density was disturbed randomly to $0.02 q\left(\delta_{\text {ran }}=0.02\right)$. Regularization parameter $\alpha$ was chosen with the use of the Morozov principle, the minimum of energy integral criterion and the Lcurve method.

Figure 2 presents the course of the energy integral and its derivative depending on the parameter $\alpha$. To solve the Cauchy problem, the authors applied such value of the regularization parameter $\alpha$ for which the energy integral $E(\alpha)$ took the minimal value, which meant that the derivative of the energy integral $E^{\prime}=\frac{d E}{d \alpha}$ reversed the sign. The value of $\alpha$ was $5.13 \times 10^{-4}$ (Table II). The Cauchy problem was also regularized for

\begin{tabular}{lccc}
\hline $\begin{array}{l}\text { Error of heat flux density } \\
\text { disturbed randomly }\end{array}$ & & $N_{\max }$ & \\
\hline$\delta_{\text {ran }}=0$ & $2.78 \cdot 10^{-2}$ & $f_{1}$ & $f_{2}$ \\
$\delta_{\text {ran }}=0.01$ & 21978535 & 0.40047 \\
$\delta_{\text {ran }}=0.02$ & 43957070 & 2814929 \\
\end{tabular}

\section{Figure 2.}

Energy integral (E) and the derivative of the energy integral $\left(E^{\prime}\right)$ depending on the value of the regularization parameter $\alpha$ (function $f_{1}$ )

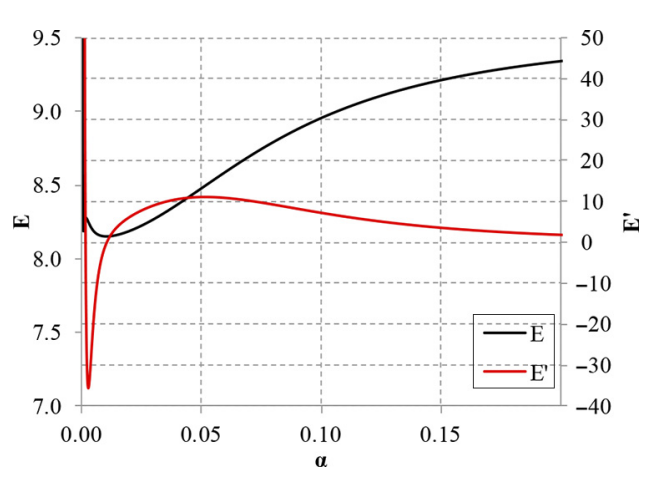


the regularization parameter $\alpha$ amounting to $4.64 \times 10^{-1}$, which was determined based on the L-curve course (Figure 3).

To choose the regularization parameter with the Morozov principle, the values of mean and maximal error $\delta_{M}$ were evaluated for the heat flux on the boundary $\Gamma_{3}$. The respective values were obtained: 0.008 and 0.02 (Table II). Next, zero of the function $F_{M}(\alpha)$ was calculated as per the equation (39). The respective values of the regularization parameter were obtained: $1.98 \times 10^{-6}$ and $4.0799 \times 10^{-2}$ (Table II).

The lowest value of the norm $N_{\max }$ amounting to $6.18 \times 10^{-2}$ (Table II) for the function $f_{1}$ was obtained for the case of choosing the regularization parameter with the use of the Morozov principle for the maximal error of the heat flux $\delta_{M}$ (Morozov_B). This criterion brought satisfying results, as did the choice of the regularization parameter made with the use of the minimum energy integral criterion $\left(N_{\max }=9.796 \times 10^{-2}\right)$. When the L-curve method was used, the obtained results were considerably worse $\left(N_{\max }=2.22 \times 10^{-1}\right)$. For the Morozov principle, for the mean error $\delta_{M}$ of the heat flux (Morozov_A), the highest value of the norm $N_{\max }$ amounting to 50.42 was obtained. Distributions of temperature on the boundary $\Gamma_{1}$ resulting from the analytical solution (AS) and from the solution of the Cauchy problem are presented in Figure 4.

\subsection{Example 2}

Calculations were made for the function $f_{2}$ [Equation (43)]. Heat flux density was disturbed randomly to $0.02 q\left(\delta_{\text {ran }}=0.02\right)$. The best results were obtained for the choice of the regularization parameter made with the use of the minimum energy integral criterion

\begin{tabular}{lcrr}
\hline $\begin{array}{l}\text { Method of the choice of the } \\
\text { regularization parameter }\end{array}$ & $\delta_{M}$ & $\alpha$ & $N_{\max }$ \\
\hline Morozov_A & 0.008 & $1.98 \times 10^{-6}$ & 50.42 \\
Morozov_B & 0.02 & $4.0799 \times 10^{-2}$ & $6.18 \times 10^{-2}$ \\
E & - & $5.13 \times 10^{-4}$ & $9.796 \times 10^{-2}$ \\
L-curve & - & $4.64 \times 10^{-1}$ & $2.22 \times 10^{-1}$ \\
\hline
\end{tabular}

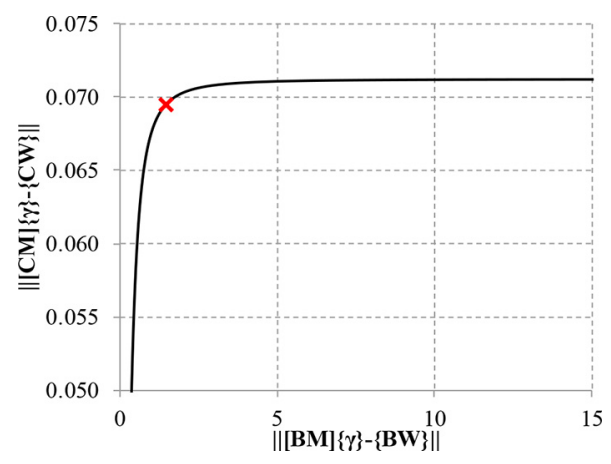

Figure 3.

L-curve with the point for which the regularization parameter $\alpha$ was chosen (function $f_{1}$ )

Table II.

Values of the measurement data error $\delta_{M}$, of the regularization parameter $\alpha$ and of the norm $N_{\max }$ for the choice of the regularization parameter $\alpha$ made using the Morozov principle

(Morozov_A and Morozov_B), the minimum of energy integral criterion $(\mathrm{E})$ and the L-curve method (L-curve) for the function $f_{1}$ 
HFF

30,10

4486

$\left(N_{\max }=4.77 \times 10^{-2}\right)$, and the worse results were obtained for the L-curve method $\left(N_{\max }=\right.$ 0.361 ). Values of the regularization parameter and of the norm $N_{\max }$, being the measure of the quality of the parameter $\alpha$ choice, for the function $f_{2}$ are summarized in Table III. Distributions of temperature on the boundary $\Gamma_{1}$ for the AS and for the solution to the Cauchy problem with regularization are presented in Figure 5. Distribution of temperature on the boundary $\Gamma_{1}$ obtained with the use of the minimum of energy integral criterion slightly diverges from the AS.

To examine thoroughly the criterion for the regularization parameter selection with the use of the minimum of energy integral, calculations were performer also for the following functions:

$$
\begin{gathered}
f_{3}=\cos x \cosh y+\sin x \sinh y \\
f_{4}=e^{x} \sin y \\
f_{5}=x^{3}-3 x y^{2}+e^{2 y} \sin 2 x-e^{x} \cos y \\
f_{6}=e^{x^{2}-y^{2}} \sin 2 x y
\end{gathered}
$$

which were chosen based on publications (Liu et al., 2018; Conde Mones et al., 2017; Fu et al., 2013; Sun, 2017). Values of the regularization parameter and of the norm $N_{\max }$ for functions $f_{3}-f_{6}$ are summarized in Table IV. For the function $f_{6}$ and the disturbance to the heat flux density $\delta_{\text {ran }}=0.02$ and $\delta_{\text {ran }}=0.05$ the minimum of energy integral was not achieved. Distributions of temperature on the boundary $\Gamma_{1}$ being sought are presented in Figure 6 . For

\section{Figure 4.}

Distribution of temperature on the boundary $\Gamma_{1}$ obtained from the AS and form the Cauchy problem when the regularization parameter $\alpha$ was chosen with the use of the Morozov principle (Morozov_B), the minimum of energy integral criterion (E) and the L-curve method (L-curve) for the function $f_{1}$

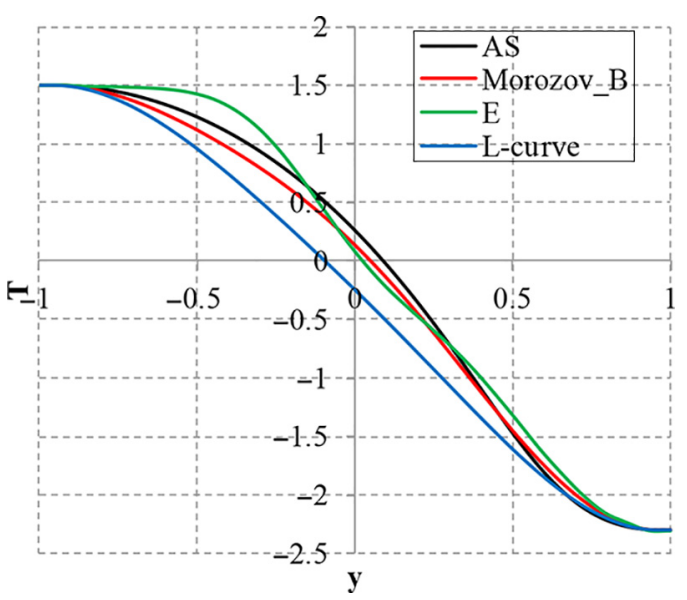


functions $f_{3}-f_{5}$ the disturbance $\delta_{\text {ran }}=0.05$ was taken into account, and for the function $f_{6}$ it was $\delta_{\text {ran }}=0.01$.

\section{Conclusion}

This paper presents the solution of the Cauchy problem for Laplace's equation. Obtained distributions of temperature on the boundary $\Gamma_{1}$ were analyzed in terms of the dependence on the method for choosing the regularization parameter. The best results were obtained for the choice of the regularization parameter made with the use of the minimum of energy integral criterion and the Morozov principle $\left(\delta_{M}\right.$ is the maximal error for the heat flux on the boundary $\Gamma_{3}$ ). The advantage of the application of the minimum energy integral criterion is a unique determination of the regularization parameter $\alpha$ for which $E(\alpha)$ has minimal value. Regularization made with the use of the minimum energy integral criterion gives satisfying results. However, its disadvantage is the fact that not for all calculation examples the minimum energy integral was determined. For the Morozov principle, the obtained

Method of the choice of the regularization parameter

Morozov_A

Morozov_B

$\mathrm{E}$

L-curve
0.004

$-$

\begin{tabular}{ccc}
$\delta_{M}$ & $\alpha$ & $N_{\max }$ \\
\hline 0.002 & $1.84 \times 10^{-3}$ & 0.115 \\
0.004 & $6.702 \times 10^{-3}$ & 0.174 \\
- & $3.37 \times 10^{-4}$ & $4.77 \times 10^{-2}$ \\
- & $3.998 \times 10^{-1}$ & 0.361
\end{tabular}

\begin{tabular}{ccc}
$\delta_{M}$ & $\alpha$ & $N_{\max }$ \\
\hline 0.002 & $1.84 \times 10^{-3}$ & 0.115 \\
0.004 & $6.702 \times 10^{-3}$ & 0.174 \\
- & $3.37 \times 10^{-4}$ & $4.77 \times 10^{-2}$ \\
- & $3.998 \times 10^{-1}$ & 0.361
\end{tabular}

\section{Cauchy problem for the Laplace equation}

4487

Table III.

Values of the measurement data

error $\delta_{M}$, of the

regularization parameter $\alpha$ and of the norm $N_{\max }$ for

the choice of the

regularization parameter $\alpha$ made using the Morozov principle

(Morozov_A and Morozov_B), the minimum of energy integral criterion (E) and of the L-curve method (L-curve) for the function $f_{2}$

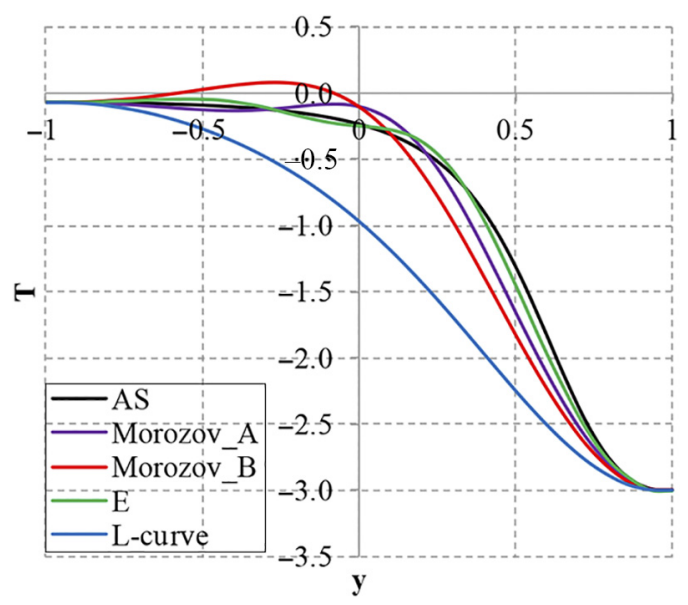

Figure 5.

Distribution of temperature on the boundary $\Gamma_{1}$ obtained from the AS and form the solution to the Cauchy problem when the regularization parameter $\alpha$ was chosen with the use of the Morozov principle

(Morozov_A and

Morozov_B), the minimum of energy integral criterion (E) and of the L-curve method (L-curve) for the function $f_{2}$ 
HFF

30,10

4488

results (distribution of temperature on the boundary $\Gamma_{1}$ ) depend on calculation or evaluation of the value of the heat flux $\delta_{M}$ error, what is a disadvantage of this method. Inexact evaluation of the measurement data error can result in obtaining the distribution of temperature on the boundary $\Gamma_{1}$, which is subject to great uncertainty. The choice of the parameter $\alpha$ made with the use of the L-curve method gave the worst results. Smooth L-curve course was obtained, what was related to the problem with the unique determination of the regularization parameter $\alpha$ using this method.

\section{Table IV.}

Values of the regularization parameter and of the norm $N_{\max }$ for functions $f_{3}-f_{6}$ with the disturbance to the heat flux density $\delta_{\text {ran }}$ from 0.01 to 0.05

\begin{tabular}{lcccccr}
\hline & \multicolumn{2}{c}{$\delta_{\text {ran }}=0.01$} & \multicolumn{2}{c}{$\delta_{\text {ran }}=0.02$} & \multicolumn{2}{c}{$\delta_{\text {ran }}=0.05$} \\
Function & $\alpha$ & $N_{\text {max }}$ & $\alpha$ & $N_{\text {max }}$ & $\alpha$ & \multicolumn{1}{c}{$N_{\text {max }}$} \\
\hline$f_{3}$ & $2.98 \times 10^{-2}$ & $2.38 \times 10^{-3}$ & $2.89 \times 10^{-2}$ & $4.49 \times 10^{-3}$ & $2.803 \times 10^{-2}$ & $1.107 \times 10^{-2}$ \\
$f_{4}$ & $4.99 \times 10^{-4}$ & $3.46 \times 10^{-2}$ & $1.0 \times 10^{-3}$ & $3.98 \times 10^{-2}$ & $1.0 \times 10^{-3}$ & $6.406 \times 10^{-2}$ \\
$f_{5}$ & $4.99 \times 10^{-4}$ & $3.46 \times 10^{-2}$ & $1.0 \times 10^{-3}$ & $3.98 \times 10^{-2}$ & $1.0 \times 10^{-2}$ & $2.16 \times 10^{-1}$ \\
$f_{6}$ & $1.0 \times 10^{-3}$ & $2.33 \times 10^{-1}$ & \multicolumn{2}{c}{ No minimum } & \multicolumn{2}{c}{ No minimum } \\
\hline
\end{tabular}

Figure 6.

Distribution of temperature on the boundary $\Gamma_{1}$ obtained from the AS and form the solution to the Cauchy problem when the regularization parameter $\alpha$ was chosen with the use of the minimum of energy integral criterion (E) for functions $f_{2}-f_{6}$
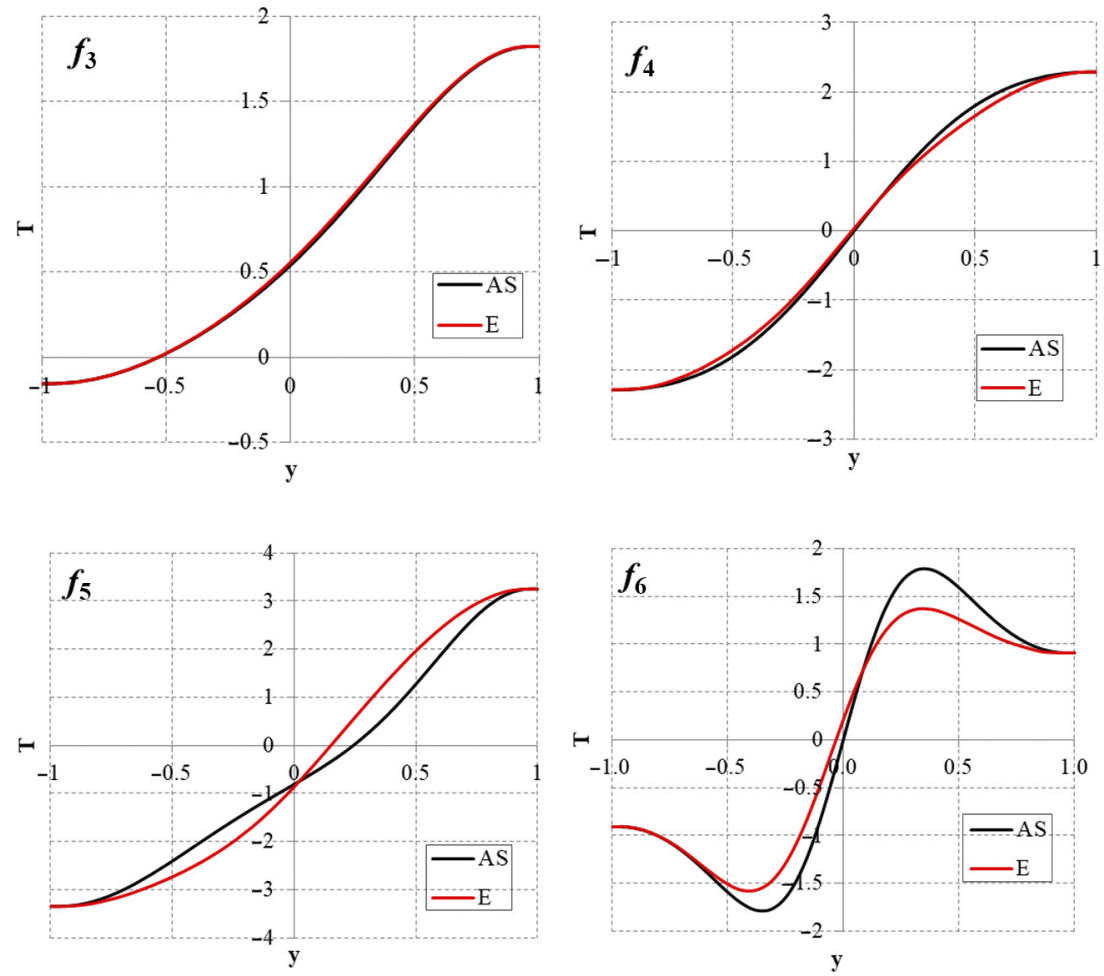


\section{References}

Beck, J.V. and Woodbury, K.A. (2016), "Inverse heat conduction problem: sensitivity coefficient insights, filter coefficients, and intrinsic verification”, International Journal of Heat and Mass Transfer, Vol. 97, pp. 578-588.

Chen, B., Sun, Y. and Zhuang, Z. (2019), "Method of fundamental solutions for a Cauchy problem of the Laplace equation in a half-plane", Boundary Value Problems, Vol. 2019 No. 1, pp. 1-14.

Cheng, H. and Feng, X.L. (2014), "A new filtering method for the Cauchy problem of the Laplace equation”, International Journal of Computer Mathematics, Vol. 91 No. 12, pp. 2621-2630.

Cheruvu, V. (2017), "Wavelet regularized solution of Laplace equation in an arbitrary shaped domain", International Journal of Applied and Computational Mathematics, Vol. 3 No. S1, pp. 775-784.

Ciałkowski, M. and Frąckowiak, A. (2002), "Solution of the stationary 2D inverse heat conduction problem by Treffetz method", Journal of Thermal Science, Vol. 11 No. 2, pp. 148-162.

Ciałkowski, M.J. and Grysa, K. (2010), "Trefftz method in solving the inverse problem", Journal of Inverse and Ill-Posed Problems, Vol. 18 No. 6, pp. 595-616.

Conde Mones, J.J., Juárez Valencia, L.H., Oliveros Oliveros, J.J. and León Velasco, D.A. (2017), "Stable numerical solution of the Cauchy problem for the Laplace equation in irregular annular regions", Numerical Methods for Partial Differential Equations, Vol. 33 No. 6, pp. 1799-1822.

Delvare, F. and Cimetière, A. (2017), "Unique discrete harmonic continuation and data completion problems using the fading regularization method", Numerical Algorithms, Vol. 75 No. 3, pp. 731-751.

Djerrar, I., Alem, L. and Chorfi, L. (2017), "Regularization method for the radially symmetric inverse heat conduction problem", Boundary Value Problems, Vol. 2017 No. 1, pp. 1-14.

Fracckowiak, A. and Ciałkowski, M. (2018), "Application of discrete Fourier transform to inverse heat conduction problem regularization", International Journal of Numerical Methods for Heat and Fluid Flow, Vol. 28 No. 1, pp. 239-253.

Frąckowiak, A., Ciałkowski, M. and Wróblewska, A. (2017), "Application of iterative algorithms for gas-turbine blades cooling optimization”, International Journal of Thermal Sciences, Vol. 118, pp. 198-206.

Frąckowiak, A., von Wolfersdorf, J. and Ciałkowski, M. (2011), "Solution of the inverse heat conduction problem described by the Poisson equation for a cooled gas-turbine blade", International Journal of Heat and Mass Transfer, Vol. 54 Nos 5/6, pp. 1236-1243.

Frąckowiak, A., von Wolfersdorf, J. and Ciałkowski, M. (2015b), “An iterative algorithm for the stable solution of inverse heat conduction problems in multiply-connected domains", International Journal of Thermal Sciences, Vol. 96, pp. 268-276.

Frąckowiak, A., von Wolfersdorf, J. and Ciałkowski, M. (2019b), "Optimization of cooling of gas turbine blades with channels filled with porous material”, International Journal of Thermal Sciences, Vol. 136, pp. 370-378.

Frąckowiak, A., Botkin, N.D., Ciałkowski, M. and Hoffmann, K.-H. (2015a), "Iterative algorithm for solving the inverse heat conduction problems with the unknown source function", Inverse Problems in Science and Engineering, Vol. 23 No. 6, pp. 1056-1071.

Frąckowiak, A., Spura, D., Gampe, U. and Ciałkowski, M. (2019a), "Determination of heat transfer coefficient in a $t$-shaped cavity by means of solving the inverse heat conduction problem", International Journal of Numerical Methods for Heat and Fluid Flow, doi: 10.1108/HFF-09-2018-0484.

Fu, C.-L., Ma, Y.-J., Cheng, H. and Zhang, Y.-X. (2013), "The a posteriori Fourier method for solving the Cauchy problem for the Laplace equation with nonhomogeneous Neumann data", Applied Mathematical Modelling, Vol. 37 Nos 14/15, pp. 7764-7777.

Gelfand, I.M. and Fomin, S.W. (1979), Calculus of Variations, PWN, Warsaw. 
HFF

30,10

Grysa, K., Maciage, A. and Pawińska, A. (2012), "Solving nonlinear direct and inverse problems of stationary heat transfer by using Trefftz functions", International Journal of Heat and Mass Transfer, Vol. 55 Nos 23/24, pp. 7336-7340.

Hadamard, J. (1902), Sur Les Problèmes Aux Dérivéespartielles et Leur Signification Physique, Princeton University Bull, pp. 49-52.

Han, H., Ling, L. and Takeuchi, T. (2011), "An energy regularization for Cauchy problems of Laplace equation in annulus domain", Communications in Computational Physics, Vol. 9 No. 4, pp. 878-896.

Hasanov, A. and Mukanova, B. (2015), "Relationship between representation formulas for unique regularized solutions of inverse source problems with final overdetermination and singular value decomposition of input-output operators", Ima Journal of Applied Mathematics, Vol. 80 No. 3, pp. 676 -696.

Hożejowska, S., Piasecka, M. and Poniewski, M.E. (2009), "Boiling heat transfer in vertical minichannels. Liquid crystal experiments and numerical investigations", International Journal of Thermal Sciences, Vol. 48 No. 6, pp. 1049-1059.

Hożejowski, L. (2016), "Trefftz method for a polynomial-based boundary identification in twodimensional Laplacian problems", Journal of Theoretical and Applied Mechanics, Vol. 54 No. 3, pp. 935-944.

Jin, B. and Zheng, Y. (2006), "A meshless method for some inverse problems associated with the Helmholtz equation", Computer Methods in Applied Mechanics and Engineering, Vol. 195 Nos 19-22, pp. 2270-2288.

Joachimiak, M. and Ciałkowski, M. (2014), "Optimal choice of integral parameter in a process of solving the inverse problem for heat equation", Archives of Thermodynamics, Vol. 35 No. 3, pp. 265-280.

Joachimiak, M. and Ciałkowski, M. (2017), "Non-linear unsteady inverse boundary problem for heat conduction equation", Archives of Thermodynamics, Vol. 38 No. 2, pp. 81-100.

Joachimiak, M. and Ciałkowski, M. (2018), "Stable solution to non-stationary inverse heat conduction equation", Archives of Thermodynamics, Vol. 39 No. 1, pp. 25-37.

Joachimiak, M., Ciałkowski, M. and Frąckowiak, A. (2019a), "Stable method for solving the Cauchy problem with the use of Chebyshev polynomials", International Journal of Numerical Methods for Heat and Fluid Flow, available at: https://doi.org/10.1108/HFF-05-2019-0416

Joachimiak, M., Frąckowiak, A. and Ciałkowski, M. (2016), "Solution of inverse heat conduction equation with the use of Chebyshev polynomials", Archives of Thermodynamics, Vol. 37 No. 4, pp. 73-88.

Joachimiak, M., Joachimiak, D., Ciałkowski, M., Małdziński, L., Okoniewicz, P. and Ostrowska, K. (2019b), "Analysis of the heat transfer for processes of the cylinder heating in the heat-treating furnace on the basis of solving the inverse problem", International Journal of Thermal Sciences, Vol. 145.

Kołodziej, J.A. and Mierzwiczak, M. (2008), "The application of the method of fundamental solutions to a simulation of the two-dimensional sloshing phenomenon", Journal of Mechanics of Materials and Structures, Vol. 3 No. 6, pp. 1087-1095.

Laneev, E.B. (2018), "Construction of a Carleman function based on the Tikhonov regularization method in an Ill-Posed problem for the Laplace equation", Differential Equations, Vol. 54 No. 4, pp. 476-485.

Lin, J., Liu, C.S., Chen, W. and Sun, L. (2018), "A novel Trefftz method for solving the multi-dimensional direct and Cauchy problems of Laplace equation in an arbitrary domain", Journal of Computational Science, Vol. 25, pp. 16-27.

Liu, C.-S. and Wang, F. (2018), "An energy method of fundamental solutions for solving the inverse Cauchy problems of the Laplace equation”, Computers and Mathematics with Applications, Vol. 75 No. 12, pp. 4405-4413.

Liu, C.-S., Wang, F. and Gu, Y. (2018), "Trefftz energy method for solving the Cauchy problem of the Laplace equation", Applied Mathematics Letters, Vol. 79, pp. 187-195. 
Maciejewska, B. and Piasecka, M. (2017), "Trefftz function-based thermal solution of inverse problem in unsteady-state flow boiling heat transfer in a minichannel", International Journal of Heat and Mass Transfer, Vol. 107, pp. 925-933.

Marin, L. (2005), "Numerical solution of the Cauchy problem for steady-state heat transfer in twodimensional functionally graded materials", International Journal of Solids and Structures, Vol. 42 No. 15, pp. 4338-4351.

Marin, L. (2010), "An alternating iterative MFS algorithm for the Cauchy problem for the modified Helmholtz equation”, Computational Mechanics, Vol. 45 No. 6, pp. 665-677.

Marin, L. (2011), "Relaxation procedures for an iterative MFS algorithm for two-dimensional steadystate isotropic heat conduction Cauchy problems", Engineering Analysis with Boundary Elements, Vol. 35 No. 3, pp. 415-429.

Marin, L. (2016), "An invariant method of fundamental solutions for two-dimensional steady-state anisotropic heat conduction problems", International Journal of Heat and Mass Transfer, Vol. 94, pp. 449-464.

Marin, L. and Munteanu, L. (2010), "Boundary reconstruction in two-dimensional steady state anisotropic heat conduction using a regularized meshless method", International Journal of Heat and Mass Transfer, Vol. 53 Nos 25/26, pp. 5815-5826.

Mierzwiczak, M. and Kołodziej, J.A. (2011), "The determination temperature-dependent thermal conductivity as inverse steady heat conduction problem", International Journal of Heat and Mass Transfer, Vol. 54 No. 4, pp. 790-796.

Mierzwiczak, M., Chen, W. and Fu, Z.-J. (2015), "The singular boundary method for steady-state nonlinear heat conduction problem with temperature-dependent thermal conductivity", International Journal of Heat and Mass Transfer, Vol. 91, pp. 205-217.

Morozov, V.A. (1984), Regularization Methods for Solving Incorrectly Posed Problems, Springer-Verlag, New York, NY.

Niu, R.P., Liu, G.R. and Li, M. (2014), "Reconstruction of dynamically changing boundary of multilayer heat conduction composite walls", Engineering Analysis with Boundary Elements, Vol. 42, pp. 92-98.

Nowak, I. (2017), "Bayesian approach applied for thermoacoustic inverse problem”, Energy, Vol. 141, pp. 2519-2527.

Paszkowski, S. (1975), Numerical Application of Multinomials and Chebyshev Series, PWN, Warsaw.

Sun, Y. (2016), "A meshless method based on the method of fundamental solution for solving the steady-state heat conduction problems", International Journal of Heat and Mass Transfer, Vol. 97, pp. 891-907.

Sun, Y. (2017), "Indirect boundary integral equation method for the Cauchy problem of the Laplace equation", Journal of Scientific Computing, Vol. 71 No. 2, pp. 469-498.

Taler, J., Weglowski, B. and Pilarczyk, M. (2017), "Monitoring of thermal stresses in pressure components using inverse heat conduction methods", International Journal of Numerical Methods for Heat and Fluid Flow, Vol. 27 No. 3, pp. 740-756.

Taler, J., Zima, W. and Jaremkiewicz, M. (2016), "Simple method for monitoring transient thermal stresses in pipelines", Journal of Thermal Stresses, Vol. 39 No. 4, pp. 386-397.

Tikhonov, A.N. and Arsenin, V.Y. (1977), Solutions of Ill-Posed Problems, Winston and Sons, Washington, DC.

Wróblewska, A., Frąckowiak, A. and Ciałkowski, M. (2015), "Regularization of the inverse heat conduction problem by the discrete Fourier transform", Inverse Problems in Science and Engineering, Vol. 24 No. 2, pp. 195 -212.

Yang, F., Fu, C. and Li, X. (2015), "A modified Tikhonov regularization method for the Cauchy problem of Laplace equation”, Acta Mathematica Scientia, Vol. 35B No. 6, pp. 1339-1348. 
HFF

30,10

4492
Yaparova, N.M. (2016), "Mathematical modelling and method for solving a parametric identification problem for self-test of measuring devices", Inverse Problems in Science and Engineering, Vol. 24 No. 1, pp. 77-91.

Zheng, G.H. and Zhang, Q.G. (2018), "Solving the backward problem for space fractional diffusion equation by a fractional Tikhonov regularization method", Mathematics and Computers in Simulation, Vol. 148, pp. 37-47.

Zhuang, H.L. and Chen, H.B. (2017), “A new highly anti-interference regularization method for ill-posed problems”, Vibroengineering Procedia, Vol. 15, pp. 128-133.

\section{Corresponding author}

Magda Joachimiak can be contacted at: magda.joachimiak@put.poznan.pl

For instructions on how to order reprints of this article, please visit our website: www.emeraldgrouppublishing.com/licensing/reprints.htm

Or contact us for further details: permissions@emeraldinsight.com 